\title{
Anonymizing Classification Data for Privacy Preservation
}

\author{
Benjamin C. M. Fung, Ke Wang, and Philip S. Yu, Fellow, IEEE
}

\begin{abstract}
Classification is a fundamental problem in data analysis. Training a classifier requires accessing a large collection of data. Releasing person-specific data, such as customer data or patient records, may pose a threat to individual's privacy. Even after removing explicit identifying information such as Name and SSN, it is still possible to link released records back to their identities by matching some combination of non-identifying attributes such as $\{S e x, Z i p, B i r t h d a t e\}$. A useful approach to combat such linking attacks, called $k$-anonymization [1], is anonymizing the linking attributes so that at least $k$ released records match each value combination of the linking attributes. Previous work attempted to find an optimal $k$-anonymization that minimizes some data distortion metric. We argue that minimizing the distortion to the training data is not relevant to the classification goal that requires extracting the structure of predication on the "future" data.

In this paper, we propose a $k$-anonymization solution for classification. Our goal is to find a $k$-anonymization, not necessarily optimal in the sense of minimizing data distortion, that preserves the classification structure. We conducted intensive experiments to evaluate the impact of anonymization on the classification on future data. Experiments on real life data show that the quality of classification can be preserved even for highly restrictive anonymity requirements.
\end{abstract}

Index Terms-Privacy protection, anonymity, security, integrity, data mining, classification, data sharing

\section{INTRODUCTION}

D ATA sharing in today globally networked systems poses a threat to individual privacy and organizational confidentiality. An example in Samarati [2] shows that linking medication records with a voter list can uniquely identify a person's name and her medical information. New privacy acts and legislations are recently enforced in many countries. In 2001, Canada launched the Personal Information Protection and Electronic Document Act [3] to protect a wide spectrum of information, e.g., age, race, income, evaluations, and even intentions to acquire goods or services. This information spans a considerable portion of many databases. Government agencies and companies have to revise their systems and practices to fully comply with this act in three years.

Consider a table $T$ about patient's information on Birthplace, Birthyear, Sex, and Diagnosis. If a description on $\{$ Birthplace, Birthyear, Sex $\}$ is so specific that not many people match it, releasing the table may lead to linking a unique record to an external record with explicit identity,

Research was supported in part by a research grant and a PGS scholarship from the Natural Sciences and Engineering Research Council of Canada.

B. C. M. Fung and K. Wang are with the School of Computing Science, Simon Fraser University, Burnaby, Canada. Email: \{bfung,wangk\}@cs.sfu.ca

P. S. Yu is with the IBM T. J. Watson Research Center, 19 Skyline Drive, Hawthorne, NY 10532. Email: psyu@us.ibm.com thus identifying the medical condition and compromising the privacy rights of the individual [2]. Suppose that the attributes Birthplace, Birthyear, Sex, and Diagnosis must be released (say, to some health research institute for research purposes). One way to prevent such linking is masking the detailed information of these attributes as follows:

1) If there is a taxonomical description for a categorical attribute (e.g., Birthplace), we can generalize a specific value description into a less specific but semantically consistent description. For example, we can generalize the cities San Francisco, San Diego, and Berkeley into the corresponding state California.

2) If there is no taxonomical description for a categorical attribute, we can suppress a value description to a "null value" denoted $\perp$. For example, we can suppress San Francisco and San Diego to the null value $\perp$ while keeping Berkeley.

3) If the attribute is a continuous attribute (e.g., Birthyear), we can discretize the range of the attribute into a small number of intervals. For example, we can replace specific Birthyear values from 1961 to 1965 with an interval [1961-1966).

By applying such masking operations, the information on $\{$ Birthplace,Birthyear,Sex $\}$ is made less specific and a person tends to match more records. For example, a male born in San Francisco in 1962 will match all records that have the values $\langle$ CA, [1961-1966), M $\rangle$; clearly not all matched records correspond to the person. Thus the masking operation makes it more difficult to tell whether an individual actually has the diagnosis in the matched records.

Protecting privacy is one goal. Making the released data useful to data analysis is another goal. In this paper, we consider classification analysis [4]. The next example shows that if masking is performed "carefully", privacy can be protected while preserving the usefulness for classification.

Example 1 (The running example): Consider the data in Table I and taxonomy trees in Figure 1. The table has 34 records in total. Each row represents one or more records with the Class column containing the class frequency of the records represented, Y for "income $>50 \mathrm{~K}$ " and $\mathrm{N}$ for "income $\leq 50 \mathrm{~K}$ ". For example, the third row represents 5 records having Education $=11$ th, Sex $=$ Male and Work_Hrs $=35$. The value $2 \mathrm{Y} 3 \mathrm{~N}$ in the Class column conveys that 2 records have the class $\mathrm{Y}$ and 3 records have the class N. Semantically, this (compressed) table is equivalent to the table containing 34 rows with each row representing one record. There is only one record for "female doctor" (the last row), which makes 


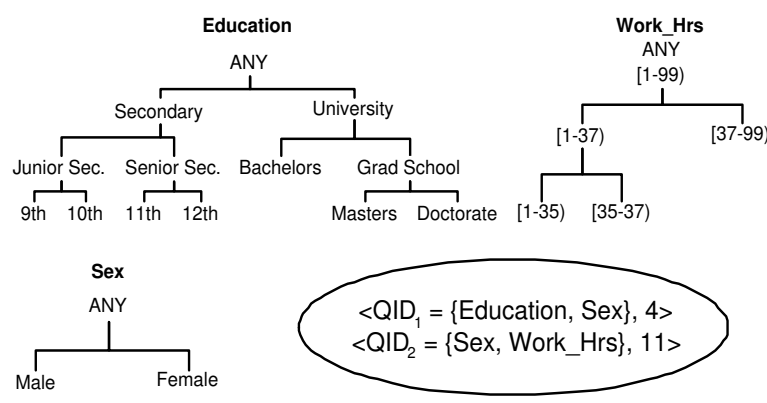

Fig. 1. Taxonomy trees and QIDs

the person represented uniquely distinguishable from others by Sex and Education. To make "female doctor" less unique, we can generalize Masters and Doctorate to Grad School. As a result, "she" becomes less distinguishable by being one of the four females with a graduate school degree. As far as classification is concerned, no information is lost in this generalization because Class does not depend on the distinction of Masters and Doctorate.

In the classification problem, a classifier is built from the training data and is used to classify the future data. It is important that the classifier makes use of the structure that will repeat in the future data, not the noises that occur only in the training data. In Table I, 19 out of 22 persons having Work_Hrs $\geq 37$ are in the class $\mathrm{Y}$, and only 3 persons having Work_Hrs $\geq 37$ are in the class N. It is not likely that this difference is entirely due to sampling noises. In contrast, $M$ and $F$ of $S e x$ seem to be arbitrarily associated with both classes, suggesting that sex cannot be used to predict his/her class.

In this paper, we consider the following $k$-anonymization for classification. The data provider wants to release a person-specific table for modelling classification of a specified class attribute in the table. Two types of information in the table are released. The first type is sensitive information, such as Diagnosis. The second type is the quasiidentifier [5] [1], which is a combination of attributes such as $\{$ Birthplace, Birthyear, Sex $\}$. The quasi-identifier does not identify individuals but can be used to link to a person if the combination is unique. The data provider wants to prevent linking the released records to an individual through the quasiidentifier. This privacy requirement is specified by the $k$ anonymity [1]: if one record in the table has some value on the quasi-identifier, at least $k-1$ other records have that value. The $k$-anonymization for classification is to produce a masked table that satisfies the $k$-anonymity requirement and retains useful information for classification. A formal statement will be given in Section II.

If classification is the goal, why does not the data provider build and publish a classifier (instead of publishing the data)? There are real-life scenarios where it is necessary to release the data. First of all, knowing that the data is used for classification does not imply that the data provider knows exactly how the recipient may analyze the data. The recipient often has application-specific bias towards building the classifier. For example, some recipient prefers accuracy while the others
TABLE I

(COMPRESSED) TABLE

\begin{tabular}{|c|c|c|c|c|}
\hline Education & Sex & Work_Hrs & Class & \# of Recs. \\
\hline 9th & $\mathrm{M}$ & 30 & $0 \mathrm{Y} 3 \mathrm{~N}$ & 3 \\
\hline 10th & $\mathrm{M}$ & 32 & 0Y4N & 4 \\
\hline 11th & M & 35 & $2 \mathrm{Y} 3 \mathrm{~N}$ & 5 \\
\hline 12th & $\mathrm{F}$ & 37 & $3 \mathrm{Y} 1 \mathrm{~N}$ & 4 \\
\hline Bachelors & $\mathrm{F}$ & 42 & $4 \mathrm{Y} 2 \mathrm{~N}$ & 6 \\
\hline Bachelors & $\mathrm{F}$ & 44 & $4 \mathrm{YON}$ & 4 \\
\hline Masters & $\mathrm{M}$ & 44 & $4 \mathrm{YON}$ & 4 \\
\hline Masters & $\mathrm{F}$ & 44 & $3 \mathrm{YON}$ & 3 \\
\hline Doctorate & $\mathrm{F}$ & 44 & $1 \mathrm{Y} 0 \mathrm{~N}$ & 1 \\
\hline \multicolumn{3}{|c|}{ Total: } & $21 \mathrm{Y} 13 \mathrm{~N}$ & 34 \\
\hline
\end{tabular}

prefer interpretability, or some prefers recall while the others prefer precision, and so on. In other cases, the recipient may not know exactly what to do before seeing the data, such as visual data mining where the human makes decisions based on certain distributions of data records at each step. Publishing the data provides the recipient a greater flexibility of data analysis.

Our insight is as follows. Typically the data contains overly specific "noises" that are harmful to classification. To construct a classifier, noises need to be generalized into patterns that are shared by more records in the same class. The data also contains "redundant structures". For example, if any of Education and Work_Hrs is sufficient for determining the class and if one of them is distorted, the class can still be determined from the other attribute. Our approach exploits such rooms provided by noises and redundant structures to mask the data without compromising the quality of classification. To this end, we propose an information metric to focus masking operations on the noises and redundant structures. We conducted intensive experiments to evaluate the impact of anonymization on the classification of future data. Below are several useful features of our approach.

- Information and privacy guided top-down refinement. Instead of masking the most specific table bottom-up, we refine masked values top-down starting from the most masked table.

- Handling different types of attributes. We handle categorical attributes with taxonomy, categorical attributes without taxonomy, and continuous attributes.

- Handling multiple quasi-identifiers. Compared to the single quasi-identifier that contains all the attributes, we enforce $k$-anonymity on only attribute sets that can be potentially used as an quasi-identifier. This approach avoids unnecessary distortion to the data.

- Scalable and anytime solution. Our method has a linear time complexity in the table size. Moreover, the user can stop the top-down refinement any time and have a table satisfying the anonymity requirement.

The notion of $k$-anonymity was first proposed in [1]. In general, a cost metric is used to measure the data distortion of anonymization. Two types of cost metric have been considered. The first type, based on the notion of minimal generalization [2] [6], is independent of the purpose of the data release. The second type factors in the purpose of the data release such as classification [7]. The goal is to find the optimal $k$-anonymization that minimizes this cost metric. In general, achieving optimal $k$-anonymization is $N P$-hard [8] 
[9]. Greedy methods were proposed in [10] [11] [12] [13] [14] [15]. Scalable algorithms (with the exponential complexity the worst-case) for finding the optimal $k$-anonymization were studied in [2] [6] [7] [16].

Our insight is that the optimal $k$-anonymization is not suitable to classification where masking structures and masking noises have different effects: the former deems to damage classification whereas the latter helps classification. It is well known in data mining and machine learning that the unmodified data, which has the lowest possible cost according to any cost metric, often has a worse classification than some generalized (i.e., masked) data. In a similar spirit, less masked data could have a worse classification than some more masked data. This observation was confirmed by our experiments. The optimal $k$-anonymization seeks to minimize the error on the training data, thus over-fits the data, subject to the privacy constraint. Neither the over-fitting nor the privacy constraint is relevant to the classification goal that seeks to minimize the error on future data.

Besides the standard setting, extensions of $k$-anonymity were also studied. [19] proposed the notion of multidimensional $k$-anonymity where data generalization is over multidimension-at-a-time, and [17] extended multidimensional generalization to anonymize data for a specific task such as classification. [20] proposed some greedy methods to achieve $k$-anonymity with cell generalization, and showed that the cell generalization generally causes less information loss than the multidimensional generalization. These masking operations allow the co-existence of a specific value and a general value, such as Bachelor and University in Table I. Such masked data will suffer from "interpretation difficulty" in the data analysis phase. For example, the exact number of bachelors cannot be determined when only some, say only 3 out of the 10 , Bachelor's are generalized to Univer sity's. If a classifier is built from such data, it is unclear which classification rule, Bachelor $\rightarrow Y$ or University $\rightarrow N$ ?, should be used to classify bachelor.

[21] measured anonymity by the l-diversity that corresponds to some notion of uncertainty of linking a quasiidentifier to a particular sensitive value. [22] [23] proposed to bound the confidence of inferring a particular sensitive value using one or more privacy templates specified by the data provider. [15] proposed some generalization methods to simultaneously achieve $k$-anonymity and bound the confidence. [24] limited the breach probability, which is similar to the notion of confidence, and allowed a flexible threshold for each individual. $k$-anonymization for data owned by multiple parties was considered in [18]. $k$-anonymization for sequential releases was studied in [25].

\section{Problem Definition}

A data provider wants to release a person-specific table $T\left(D_{1}, \ldots, D_{m}\right.$, Class $)$ to the public for modelling the class label Class. Each $D_{i}$ is either a categorical or a continuous attribute. A record has the form $\left\langle v_{1}, \ldots, v_{m}, c l s\right\rangle$, where $v_{i}$ is a domain value for $D_{i}$ and $c l s$ is a class for Class. att $(v)$ denotes the attribute of a value $v$. The data provider also wants to protect against linking an individual to sensitive information either within or outside $T$ through some identifying attributes, called a quasi-identifier or simply QID. A sensitive linking occurs if some value of the quasi-identifier identifies a "small" number of records in $T$. This requirement is formally defined below.

Definition 1 (Anonymity requirement): Consider $p$ quasiidentifiers $Q I D_{1}, \ldots, Q I D_{p}$ on T. $a\left(q i d_{i}\right)$ denotes the number of data records in $T$ that share the value $q i d_{i}$ on $Q I D_{i}$. The anonymity of $Q I D_{i}$, denoted $A\left(Q I D_{i}\right)$, is the smallest $a\left(q i d_{i}\right)$ for any value $q i d_{i}$ on $Q I D_{i}$. A table $T$ satisfies the anonymity requirement $\left\{\left\langle Q I D_{1}, k_{1}\right\rangle, \ldots,\left\langle Q I D_{p}, k_{p}\right\rangle\right\}$ if $A\left(Q I D_{i}\right) \geq k_{i}$ for $1 \leq i \leq p$, where $k_{i}$ is the anonymity threshold on $Q I D_{i}$ specified by the data provider.

It is not hard to see that if $Q I D_{j}$ is a subset of $Q I D_{i}$, $A\left(Q I D_{i}\right) \leq A\left(Q I D_{j}\right)$. Therefore, if $k_{j} \leq k_{i}, A\left(Q I D_{i}\right) \geq k_{i}$ implies $A\left(Q I D_{j}\right) \geq k_{j}$, and $\left\langle Q I D_{j}, k_{j}\right\rangle$ can be removed in the presence of $\left\langle Q I D_{i}, k_{i}\right\rangle$. Following a similar argument, to prevent a linking through any QID that is any subset of attributes in $Q I D_{1} \cup \cdots \cup Q I D_{p}$, the single QID requirement $\langle Q I D, k\rangle$ where $Q I D=Q I D_{1} \cup \cdots \cup Q I D_{p}$ and $k=\max \left\{k_{j}\right\}$ can be specified. However, a table satisfying $\left\{\left\langle Q I D_{1}, k_{1}\right\rangle, \ldots,\left\langle Q I D_{p}, k_{p}\right\rangle\right\}$ does not have to satisfy $\langle Q I D, k\rangle$.

Example 2: Suppose that a data provider wants to release Table I. To protect linking \{Education, Sex $\}$ to sensitive information, the data provider specifies $\left\langle Q I D_{1}=\right.$ $\{$ Education, Sex $\}, 4\rangle$. This requirement is violated by $\langle 9$ th, $M\rangle,\langle$ Masters, $F\rangle$, $\langle$ Doctorate, $F\rangle$. To protect linking through $\{$ Sex,Work_Hrs $\}$ as well, the data provider can specify the two QIDs in Figure 1. To prevent linking through any combination of the identifying attributes, the data provider can specify $Q I D=\{$ Education, Sex, Work_Hrs $\}$.

Definition 1 generalizes the classic notion of $k$-anonymity by allowing multiple QIDs (with possibly different thresholds). Suppose that the data provider wants to release a table $T(A, B, C, D, S)$, where $S$ is the sensitive attribute, and knows that the recipient has access to previously released tables $T 1(A, B, X)$ and $T 2(C, D, Y)$, where $X$ and $Y$ are attributes not in $T$. To prevent linking the records in $T$ to $X$ or $Y$, the data provider only has to specify the $k$-anonymity on $Q I D 1=\{A, B\}$ and $Q I D 2=\{C, D\}$. In this case, enforcing the $k$-anonymity on $Q I D=\{A, B, C, D\}$ will distort the data more than what is necessary. All previous works suffer from this problem because they handled multiple QIDs through the single QID made up of all attributes in the multiple QIDs.

To transform $T$ to satisfy the anonymity requirement, we consider three types of masking operations on the attributes $D_{j}$ in $\cup Q I D_{i}$.

\section{Masking operations:}

1) Generalize $D_{j}$ if $D_{j}$ is a categorical attribute with a taxonomy tree. A leaf node represents a domain value and a parent node represents a less specific value. Figure 2 shows a taxonomy tree for Education. A generalized $D_{j}$ can be viewed as a "cut" through its taxonomy tree. A cut of a tree is a subset of values in the tree, denoted $C u t_{j}$, that contains exactly one value on each root-toleaf path. This type of generalization does not suffer 
from the interpretation difficulty discussed in Section I, and it was previously employed in [7] [12] [13] [14] [18] [25].

2) Suppress $D_{j}$ if $D_{j}$ is a categorical attribute with no taxonomy tree. The suppression of a value on $D_{j}$ means replacing all occurrences of the value with the special value $\perp_{j}$. All suppressed values on $D_{j}$ are represented by the same $\perp_{j}$, which is treated as a new value in $D_{j}$ by a classification algorithm. We use $S u p_{j}$ to denote the set of values suppressed by $\perp_{j}$. This type of suppression is at the value level in that $S u p_{j}$ is in general a subset of the values in the attribute $D_{j}$.

3) Discretize $D_{j}$ if $D_{j}$ is a continuous attribute. The discretization of a value $v$ on $D_{j}$ means replacing all occurrences of $v$ with an interval containing the value. Our algorithm dynamically grows a taxonomy tree for intervals at runtime, where each node represents an interval, and each non-leaf node has two child nodes representing some "optimal" binary split of the parent interval. More details will be discussed in Section III. A discretized $D_{j}$ can be represented by the set of intervals, denoted $I n t_{j}$, corresponding to the leaf nodes in the dynamically grown taxonomy tree of $D_{j}$.

Definition 2 (Anonymity for Classification): Given a table $T$, an anonymity requirement $\left\{\left\langle Q I D_{1}, k_{1}\right\rangle, \ldots,\left\langle Q I D_{p}, k_{p}\right\rangle\right\}$, and an optional taxonomy tree for each categorical attribute contained in $\cup Q I D_{i}$, mask $T$ on the attributes $\cup Q I D_{i}$ to satisfy the anonymity requirement while preserving the classification structure in the data (that is, the masked table remains useful for classifying the Class column).

The cost metric for our anonymization should be measured by the classification error on the future data. It does not work to replace this cost metric by the classification error on the masked table because a perfect classifier for the masked table (say, a classifier based on a system-assigned record ID) can be inaccurate for the future data. For this reason, our problem does not have a closed form cost metric, and an "optimal" solution to our problem is not necessarily an optimal $k$-anonymization based on a closed form cost metric, and vice versa. Therefore, the previous optimal $k$-anonymization approaches [7] [16] based on a closed-form cost metric are not suitable. A more reasonable approach is minimally, not always optimally, masking the data, with a focus on classification. We will present such an approach in Section III.

It is impractical to enumerate all masked tables because the number of masked tables can be very large. For a categorical attribute with a taxonomy tree $Y$, the number of possible cuts, denoted $C(Y)$, is equal to $C\left(Y_{1}\right) \times \ldots \times C\left(Y_{u}\right)+1$ where $Y_{1}, \ldots, Y_{u}$ are the subtrees rooted at the children of the root of $Y$ and 1 is for the trivial cut at the root of $Y . C(Y)$ increases very quickly as we unfold the definition for each subtree $Y_{i}$ recursively. For a categorical attribute without a taxonomy tree and with $q$ distinct values, there are $2^{q}$ possible suppressions because each distinct value can be either suppressed or not. For a continuous attribute, each existing value can be a potential split in the dynamically grown taxonomy tree. The number of possible masked tables is equal to the product of such numbers for all the attributes in $\cup Q I D_{i}$.
A masked table $T$ can be represented by $\left\langle\cup C u t_{j}, \cup S u p_{j}, \cup I n t_{j}\right\rangle$, where $C u t_{j}$, Sup $_{j}$, Int $_{j}$ are defined as above. If the masked $T$ satisfies the anonymity requirement, $\left\langle\cup C u t_{j}, \cup S u p_{j}, \cup I n t_{j}\right\rangle$ is called a solution set.

\section{SEARCH CRITERIA}

A table $T$ can be masked by a sequence of refinements starting from the most masked state in which each attribute is either generalized to the top most value, or suppressed to the special value $\perp$, or represented by a single interval. Our method iteratively refines a masked value selected from the current set of cuts, suppressed values and intervals, until violating the anonymity requirement. Each refinement increases the information and decreases the anonymity since records with specific values are more distinguishable. The key is selecting the "best" refinement at each step with both impacts considered.

\section{A. Refinement}

Below, we formally describe the notion of refinement on different types of attributes $D_{j} \in \cup Q I D_{i}$ and define a selection criterion for a single refinement.

Refinement for generalization. Consider a categorical attribute $D_{j}$ with a user-specified taxonomy tree. Let $\operatorname{child}(v)$ be the set of child values of $v$ in a user-specified taxonomy tree. A refinement, written $v \rightarrow \operatorname{child}(v)$, replaces the parent value $v$ with the child value in $\operatorname{child}(v)$ that generalizes the domain value in each (generalized) record that contains $v$.

Refinement for suppression. For a categorical attribute $D_{j}$ without taxonomy tree, a refinement $\perp_{j} \rightarrow\left\{v, \perp_{j}\right\}$ refers to disclosing one value $v$ from the set of suppressed values $S u p_{j}$. Let $R_{\perp_{j}}$ denote the set of suppressed records that currently contain $\perp_{j}$. Disclosing $v$ means replacing $\perp_{j}$ with $v$ in all records in $R_{\perp_{j}}$ that originally contain $v$.

Refinement for discretization. For a continuous attribute, refinement is similar to that for generalization except that no prior taxonomy tree is given and the taxonomy tree has to be grown dynamically in the process of refinement. Initially, the interval that covers the full range of the attribute forms the root. The refinement on an interval $v$, written $v \rightarrow \operatorname{child}(v)$, refers to the optimal split of $v$ into two child intervals $\operatorname{child}(v)$ that maximizes the information gain. The anonymity is not used for finding a split good for classification. This is similar to defining a taxonomy tree where the main consideration is how the taxonomy best describes the application. Due to this extra step of identifying the optimal split of the parent interval, we treat continuous attributes separately from categorical attributes with taxonomy trees.

A refinement is valid (with respect to $T$ ) if $T$ satisfies the anonymity requirement after the refinement. A refinement is beneficial (with respect to $T$ ) if more than one class is involved in the refined records. A refinement is performed only if it is both valid and beneficial. Therefore, a refinement guarantees that every newly generated qid has $a($ qid $) \geq k$.

Example 3: Continue with Example 2. Figure 2 shows a cut, indicated by the dashed curve. This cut is the lowest (maximal) in the sense that any refinement on Junior Sec. or 


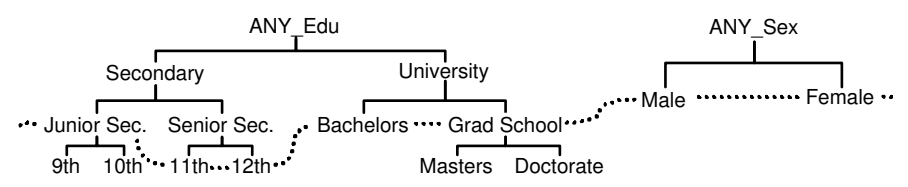

Fig. 2. Example 3: A cut for $Q I D_{1}=\{$ Education, Sex $\}$

Grad School would violate the anonymity requirement, i.e., invalid. Also, refinement on Junior Sec. or Grad School is non-beneficial since none of them refines data records in different classes.

\section{B. Selection Criterion}

We propose a selection criterion for guiding our top-down refinement process to heuristically maximize the classification goal. Consider a refinement $v \rightarrow \operatorname{child}(v)$ where $v \in D_{j}$ and $D_{j}$ is a categorical attribute with a user-specified taxonomy tree or $D_{j}$ is a continuous attribute with a dynamically grown taxonomy tree. The refinement has two effects: it increases the information of the refined records with respect to classification, and it decreases the anonymity of the refined records with respect to privacy. These effects are measured by "information gain" and denoted InfoGain( $v)$, and "anonymity loss" and denoted AnonyLoss $(v) . v$ is a good candidate for refinement if InfoGain( $v)$ is large and AnonyLoss $(v)$ is small. Our selection criterion is choosing the candidate $v$, for the next refinement, that has the maximum information-gain/anonymityloss trade-off, defined as

$$
\operatorname{Score}(v)=\frac{\operatorname{InfoGain}(v)}{\operatorname{Anony\operatorname {Loss}(v)+1} .}
$$

1 is added to AnonyLoss $(v)$ to avoid division by zero. Each choice of InfoGain $(v)$ and AnonyLoss $(v)$ gives a trade-off between classification and anonymization. It should be noted that Score is not a goodness metric of $k$-anonymization. In fact, it is difficult to have a closed form metric to capture the classification goal (on future data). We achieve this goal through this heuristic selection criterion.

For concreteness, we borrow Shannon's information theory to measure information gain [26]. Let $R_{v}$ denote the set of records masked to the value $v$ and let $R_{c}$ denote the set of records masked to a child value $c$ in $\operatorname{child}(v)$ after refining $v$. Let $|x|$ be the number of elements in a set $x .\left|R_{v}\right|=\sum_{c}\left|R_{c}\right|$, where $c \in \operatorname{child}(v)$.

$\operatorname{InfoGain}(\mathbf{v})$ : defined as

$$
\operatorname{InfoGain}(v)=I\left(R_{v}\right)-\sum_{c} \frac{\left|R_{c}\right|}{\left|R_{v}\right|} I\left(R_{c}\right),
$$

where $I\left(R_{x}\right)$ is the entropy of $R_{x}$ [26]:

$$
I\left(R_{x}\right)=-\sum_{c l s} \frac{\operatorname{freq}\left(R_{x}, \text { cls }\right)}{\left|R_{x}\right|} \times \log _{2} \frac{\operatorname{freq}\left(R_{x}, \text { cls }\right)}{\left|R_{x}\right|} .
$$

$\operatorname{freq}\left(R_{x}, \mathrm{cls}\right)$ is the number of data records in $R_{x}$ having the class $c l s$. Intuitively, $I\left(R_{x}\right)$ measures the entropy (or "impurity") of classes in $R_{x}$. The more dominating the majority class in $R_{x}$ is, the smaller $I\left(R_{x}\right)$ is (i.e., less entropy in $R_{x}$ ). Therefore, $I\left(R_{x}\right)$ measures the error because non-majority classes are considered as errors. InfoGain $(v)$ then measures the reduction of entropy after refining $v$. InfoGain $(v)$ is nonnegative. For more details on information gain and classification, see [27].

AnonyLoss(v): defined as

$$
\operatorname{AnonyLoss}(v)=\operatorname{avg}\left\{A\left(Q I D_{j}\right)-A_{v}\left(Q I D_{j}\right)\right\},
$$

where $A\left(Q I D_{j}\right)$ and $A_{v}\left(Q I D_{j}\right)$ represent the anonymity before and after refining $v \operatorname{avg}\left\{A\left(Q I D_{j}\right)-A_{v}\left(Q I D_{j}\right)\right\}$ is the average loss of anonymity for all $Q I D_{j}$ that contain the attribute of $v$.

If $D_{j}$ is a categorical attribute without taxonomy tree, the refinement $\perp_{j} \rightarrow\left\{v, \perp_{j}\right\}$ means refining $R_{\perp_{j}}$ into $R_{v}$ and $R_{\perp_{j}}^{\prime}$, where $R_{\perp_{j}}$ denotes the set of records containing $\perp_{j}$ before the refinement, $R_{v}$ and $R_{\perp_{j}}^{\prime}$ denote the set of records containing $v$ and $\perp_{j}$ after the refinement, respectively. We employ the same $\operatorname{Score}(v)$ function to measure the goodness of the refinement $\perp_{j} \rightarrow\left\{v, \perp_{j}\right\}$, except that $\operatorname{InfoGain}(v)$ is now defined as:

$$
\operatorname{InfoGain}(v)=I\left(R_{\perp_{j}}\right)-\frac{\left|R_{v}\right|}{\left|R_{\perp_{j}}\right|} I\left(R_{v}\right)-\frac{\left|R_{\perp_{j}}^{\prime}\right|}{\left|R_{\perp_{j}}\right|} I\left(R_{\perp_{j}}^{\prime}\right) \text {. }
$$

Example 4: The refinement on $A N Y \_E d u$ refines the 34 records into 16 records for Secondary and 18 records for University. The calculation of Score $\left(A N Y \_E d u\right)$ is:

$$
\begin{aligned}
& I\left(R_{A N Y \_E d u}\right)=-\frac{21}{34} \times \log _{2} \frac{21}{34}-\frac{13}{34} \times \log _{2} \frac{13}{34}=0.9597 \\
& I\left(R_{\text {Secondary }}\right)=-\frac{5}{16} \times \log _{2} \frac{5}{16}-\frac{11}{16} \times \log _{2} \frac{11}{16}=0.8960 \\
& I\left(R_{\text {University }}\right)=-\frac{16}{18} \times \log _{2} \frac{16}{18}-\frac{2}{18} \times \log _{2} \frac{2}{18}=0.5033 \\
& \text { InfoGain }\left(A N Y \_E d u\right)=I\left(R_{A N Y \_E d u}\right) \\
& \quad-\left(\frac{16}{34} \times I\left(R_{\text {Secondary }}\right)+\frac{18}{34} \times I\left(R_{U \text { niversity }}\right)\right)=0.2716 \\
& \text { AnonyLoss }\left(A N Y \_E d u\right)=(34-16) / 1=18 \\
& \text { Score }\left(A N Y \_E d u\right)=\frac{0.2716}{18+1}=0.0143 .
\end{aligned}
$$

\section{InfoGain vs. Score}

An alternative to Score is using InfoGain alone, that is, maximizing the information gain produced by a refinement without considering the loss of anonymity. This alternative may pick a candidate that has a large reduction in anonymity, which may lead to a quick violation of the anonymity requirement, thereby, prohibiting refining the data to a lower granularity. The next example illustrates this point.

Example 5: Consider Table II(a), the anonymity requirement

$\langle Q I D=\{$ Education, Sex, Work_Hrs $\}, 4\rangle$, the most masked table containing one row $\left\langle A N Y \_E d u, A N Y_{-} S e x,[1-99)\right\rangle$ with the class frequency $20 Y 20 \mathrm{~N}$, and three candidate refinements:

$A N Y_{-} E d u \rightarrow\{8 t h, 9 t h, 10 t h\}, A N Y_{-} S e x \rightarrow\{M, F\}$, and $[1-99) \rightarrow\{[1-40),[40-99)\}$.

Table II(b) shows the calculated InfoGain, AnonyLoss, and Score of the three candidate refinements. According to the InfoGain criterion, $A N Y \_E d u$ will be first refined because it has the highest InfoGain. The result is shown in Table II(c) with $A(Q I D)=4$. After that, there is no further valid refinement because refining either $A N Y \_S e x$ or $[1-99)$ will result in a violation of 4-anonymity. Note that the first 24 records in the table fail to separate the $4 \mathrm{~N}$ from the other $20 \mathrm{Y}$. 
TABLE II

COMPARING In foGain AND Score FOR EXAMPLE 5

(a) (Compressed) table

\begin{tabular}{|c|c|c|c|c|}
\hline Education & Sex & Work_Hrs & Class & \# of Recs. \\
\hline 10th & M & 40 & $20 \mathrm{Y0N}$ & 20 \\
\hline 10th & M & 30 & 0Y4N & 4 \\
\hline 9th & $\mathrm{M}$ & 30 & $0 \mathrm{Y} 2 \mathrm{~N}$ & 2 \\
\hline 9th & $\mathrm{F}$ & 30 & 0Y4N & 4 \\
\hline 9th & $\mathrm{F}$ & 40 & 0Y6N & 6 \\
\hline 8th & $\mathrm{F}$ & 30 & $0 \mathrm{Y} 2 \mathrm{~N}$ & 2 \\
\hline 8 th & $\mathrm{F}$ & 40 & $0 \mathrm{Y} 2 \mathrm{~N}$ & 2 \\
\hline & & Total: & $20 \mathrm{Y} 20 \mathrm{~N}$ & 40 \\
\hline
\end{tabular}

(b) Statistics for the most masked table

\begin{tabular}{|c|c|c|c|}
\hline Candidate & InfoGain & AnonyLoss & Score \\
\hline ANY_Edu & 0.6100 & $40-4=36$ & $0.6100 /(36+1)=0.0165$ \\
\hline ANY_Sex & 0.4934 & $40-14=26$ & $0.4934 /(26+1)=0.0183$ \\
\hline [1-99) & 0.3958 & $40-12=28$ & $0.3958 /(28+1)=0.0136$ \\
\hline
\end{tabular}

(c) Final masked table by In foGain

\begin{tabular}{|c|c|c|c|c|}
\hline Education & Sex & Work_Hrs & Class & \# of Recs. \\
\hline 10th & ANY_Sex & {$[1-99)$} & 20Y4N & 24 \\
\hline 9th & ANY_Sex & {$[1-99)$} & 0Y12N & 12 \\
\hline 8th & ANY_Sex & {$[1-99)$} & 0Y4N & 4 \\
\hline
\end{tabular}

(d) Intermediate masked table by Score

\begin{tabular}{|c|c|c|c|c|}
\hline Education & Sex & Work_Hrs & Class & \# of Recs. \\
\hline ANY_Edu & M & {$[1-99)$} & $20 Y 6 N$ & 26 \\
\hline ANY_Edu & F & {$[1-99)$} & 0Y14N & 14 \\
\hline
\end{tabular}

(e) Final masked table by Score

\begin{tabular}{|c|c|c|c|c|}
\hline Education & Sex & Work_Hrs & Class & \# of Recs. \\
\hline ANY_Edu & M & {$[40-99)$} & 20Y0N & 20 \\
\hline ANY_Edu & M & {$[1-40)$} & 0Y6N & 6 \\
\hline ANY_Edu & F & {$[40-99)$} & 0Y8N & 8 \\
\hline ANY_Edu & F & {$[1-40)$} & 0Y6N & 6 \\
\hline
\end{tabular}

In contrast, according to the Score criterion, $A N Y \_S e x$ will be first refined. The result is shown in Table II(d), and $A(Q I D)=14$. Subsequently, further refinement on $A N Y \_E d u$ is invalid because it will result in $a(\langle 9 t h, M,[1-$ $99)\rangle)=2<k$, but the refinement on $[1-99)$ is valid because it will result in $A(Q I D)=6 \geq k$. The final masked table is shown in Table II(e) where the information for separating the two classes is preserved. Thus by considering the information/anonymity trade-off, the $S$ core criterion produces a more desirable sequence of refinements for classification.

\section{TOP-DOWN REFINEMENT}

\section{A. The Algorithm}

We present our algorithm, Top-Down Refinement (TDR). In a preprocessing step, we compress the given table $T$ by removing all attributes not in $\cup Q I D_{i}$ and collapsing duplicates into a single row with the Class column storing the class frequency as in Table I. The compressed table is typically much smaller than the original table. Below, the term "data records" refers to data records in this compressed form. There exists a masked table satisfying the anonymity requirement if and only if the most masked table does, i.e., $|T| \geq k$. This condition is checked in the preprocessing step as well. To focus on main ideas, we assume that $|T| \geq k$ and the compressed table fits in the memory. Section IV-E, we will discuss the modification needed if the compressed table does not fit in the memory.

High level description of our algorithm. Algorithm 1 summarizes the conceptual algorithm. Initially, $\mathrm{Cut}_{j}$ contains

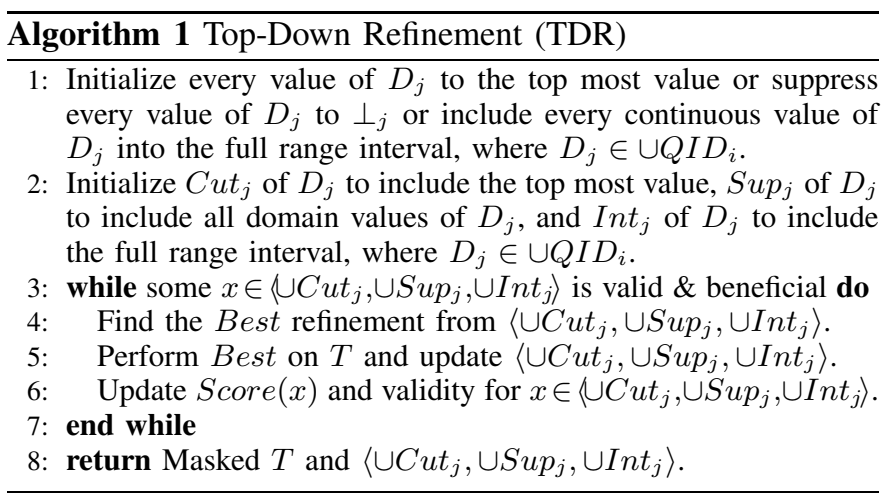

only the top most value for a categorical attribute $D_{j}$ with a taxonomy tree, $S u p_{j}$ contains all domain values of a categorical attribute $D_{j}$ without a taxonomy tree, and $I n t_{j}$ contains the full range interval for a continuous attribute $D_{j}$. The valid, beneficial refinements in $\left\langle\cup C u t_{j}, \cup S u p_{j}, \cup I n t_{j}\right\rangle$ form the set of candidates. At each iteration, we find the candidate of the highest Score, denoted Best (Line 4), apply Best to $T$ and update $\left\langle\cup C u t_{j}, \cup S u p_{j}, \cup I_{n} t_{j}\right\rangle$ (Line 5 ), and update Score and the validity of the candidates in $\left\langle\cup C u t_{j}, \cup S u p_{j}, \cup I_{n} t_{j}\right\rangle$ (Line 6). The algorithm terminates when there is no more candidate in $\left\langle\cup C u t_{j}, \cup S u p_{j}, \cup \operatorname{Int}_{j}\right\rangle$, in which case it returns the masked table together with the solution set $\left\langle\cup C u t_{j}, \cup S u p_{j}, \cup \operatorname{Int}_{j}\right\rangle$.

Example 6: Consider the anonymity requirement:

$$
\begin{aligned}
\left\{\left\langleQ I D_{1}\right.\right. & =\{\text { Education, Sex }\}, 4\rangle, \\
\left\langle Q I D_{2}\right. & =\{\text { Sex }, \text { Work_Hrs }\}, 11\rangle\} .
\end{aligned}
$$

Assume that the taxonomy trees in Figure 1 are specified for Education and Sex. Initially, all data records in Table I are masked and collapsed into a single row $\left\langle A N Y Y_{\_} E d u, A N Y_{-} S e x,[1-99)\right\rangle$, with the class frequency $21 \mathrm{Y} 13 \mathrm{~N}$ and $\cup C u t_{j}=\left\{A N Y_{-} E d u, A N Y_{-} S e x\right\}$ and $\cup I_{n} t_{j}=\{[1-99)\}$. All refinements in $\left\langle\cup C u t_{j}, \cup I n t_{j}\right\rangle$ are candidates. To find the best refinement, we need to compute Score $\left(A N Y \_E d u\right)$, Score $\left(A N Y \_S e x\right)$, Score $([1-99))$.

Our algorithm obtains the masked $T$ by iteratively refining the table from the most masked state. An important property of TDR is that the anonymity requirement is anti-monotone with respect to the top-down refinement: if it is violated before a refinement, it remains violated after the refinement. This is because a refinement never equates distinct values, therefore, never increases the count of duplicates, a(qid). Hence, the hierarchically organized search space with the most masked state at the top is separated by a border above which lie all satisfying states and below which lie all violating states. The top-down refinement finds a state on the border and this state is maximally refined in that any further refinement of it would cross the border and violate the anonymity requirement. Note that there may be more than one maximally refined state on the border. Our algorithm finds the one based on the heuristic selection criterion of maximizing Score at each step. Samarati [2] presents some results related to anti-monotonicity, but the results are based on a different masking model that generalizes all values in an attribute to the same level and suppresses data at the record level. 
Theorem 1: Algorithm 1 finds a maximally refined table that satisfies the given anonymity requirement.

Algorithm 1 makes no claim on efficiency. In fact, in a straightforward implementation, Line 4,5 and 6 require scanning all data records and recomputing Score for all candidates in $\left\langle\cup C u t_{j}, \cup S u p_{j}, \cup I n t_{j}\right\rangle$. Obviously, this is not scalable. The key to the efficiency of our algorithm is $d i$ rectly accessing the data records to be refined, and updating Score based on some statistics maintained for candidates in $\left\langle\cup C u t_{j}, \cup S u p_{j}, \cup I n t_{j}\right\rangle$. In the rest of this section, we explain a scalable implementation of Line 4, 5 and 6.

\section{B. Find the Best Refinement (Line 4)}

This step makes use of computed InfoGain $(x)$ and $A_{x}\left(Q I D_{i}\right)$ for all candidates $x$ in $\left\langle\cup C u t_{j}, \cup S u p_{j}, \cup I_{n} t_{j}\right\rangle$ and computed $A\left(Q I D_{i}\right)$ for each $Q I D_{i}$. Before the first iteration, such information is computed in an initialization step for every top most value, every suppressed value, and every full range interval. For each subsequent iteration, such information comes from the update in the previous iteration (Line 6). Finding the best refinement Best involves at most $\left|\cup C u t_{j}\right|+\left|\cup S u p_{j}\right|+\left|\cup I n t_{j}\right|$ computations of Score without accessing data records. Updating InfoGain $(x)$ and $A_{x}\left(Q I D_{i}\right)$ will be considered in Section IV-D.

\section{Perform the Best Refinement (Line 5)}

We consider two cases of performing the Best refinement, corresponding to whether a taxonomy tree is available for the attribute $D_{j}$ for Best.

Case 1: $D_{j}$ has a taxonomy tree. Consider the refinement Best $\rightarrow \operatorname{child}($ Best $)$, where Best $\in D_{j}$, and $D_{j}$ is either a categorical attribute with a specified taxonomy tree or a continuous attribute with a dynamically grown taxonomy tree. First, we replace Best with $\operatorname{child(Best)}$ in $\left\langle\cup C u t_{j}, \cup I n t_{j}\right\rangle$. Then, we need to retrieve $R_{B e s t}$, the set of data records masked to Best, to tell the child value in $\operatorname{child}(B e s t)$ for each individual data record. We present a data structure, Taxonomy Indexed PartitionS (TIPS), to facilitate this operation. This data structure is also crucial for updating $\operatorname{InfoGain}(x)$ and $A_{x}\left(Q I D_{i}\right)$ for candidates $x$. The general idea is to group data records according to their masked records on $\cup Q I D_{i}$.

Definition 3 (TIPS): TIPS is a tree structure with each node representing a masked record over $\cup Q I D_{i}$, and each child node representing a refinement of the parent node on exactly one attribute. Stored with each leaf node is the set of (compressed) data records having the same masked record, called a leaf partition. For each candidate refinement $x, P_{x}$ denotes a leaf partition whose masked record contains $x$, and $\operatorname{Link}_{x}$ denotes the link of all such $P_{x}$. The head of $\operatorname{Link}_{x}$ is stored with $x$.

The masked table is represented by the leaf partitions of TIPS. Link $k_{x}$ provides a direct access to $R_{x}$, the set of (original) data records masked by the value $x$. Initially, TIPS has only one leaf partition containing all data records, masked by the top most value or interval on every attribute in $\cup Q I D_{i}$. In each iteration, we perform the best refinement Best by refining the leaf partitions on $L i n k_{B e s t}$.
Refine Best in TIPS. We refine each leaf partition $P_{\text {Best }}$ found on Link $k_{\text {Best }}$ as follows. For each value $c$ in child(Best), a child partition $P_{c}$ is created under $P_{B e s t}$, and data records in $P_{\text {Best }}$ are split among the child partitions: $P_{c}$ contains a data record in $P_{B e s t}$ if a categorical value $c$ generalizes the corresponding domain value in the record, or if an interval $c$ contains the corresponding domain value in the record. An empty $P_{c}$ is removed. $L i n k_{c}$ is created to link up all $P_{c}$ 's for the same $c$. Also, link $P_{c}$ to every $\operatorname{Lin} k_{x}$ to which $P_{\text {Best }}$ was previously linked, except for Link $k_{\text {Best }}$. Finally, mark $c$ as "beneficial" if $R_{c}$ has more than one class, where $R_{c}$ denotes the set of data records masked to $c$.

This is the only operation that actually accesses data records in the whole algorithm. The overhead is maintaining Link $k_{x}$. For each attribute in $\cup Q I D_{i}$ and each leaf partition on $\operatorname{Link}_{B e s t}$, there are at most $\mid$ child(Best)| "relinkings". Therefore, there are at most $\left|\cup Q I D_{i}\right| \times\left|\operatorname{Link}_{\text {Best }}\right| \times \mid \operatorname{child}($ Best $) \mid$ "relinkings" for applying Best.

Example 7: Continue with Example 6. Initially, TIPS has only one leaf partition containing all data records and representing the masked record $\left\langle A N Y \_E d u, A N Y_{-}\right.$Sex,$\left.[1-99)\right\rangle$. Let the best refinement be $[1-99) \rightarrow\{[1-37),[37-99)\}$ on Work_Hrs. We create two child partitions under the root partition as in Figure 3, and split data records between them. Both child partitions are on $\operatorname{Link}_{A N Y_{-} E d u}$ and $\operatorname{Link}_{A N Y_{-} S e x}$. $\cup$ Int $_{j}$ is updated into $\{[1-37),[37-99)\}$ and $\cup C u t_{j}$ remains unchanged. Suppose that the next best refinement is $A N Y_{\_} E d u \rightarrow\{$ Secondary, University $\}$, which refines the two leaf partitions on $L_{i n k_{A N Y} E d u}$, resulting in the TIPS in Figure 3.

Count statistics in TIPS. A scalable feature of our algorithm is maintaining some statistical information for each candidate $x$ in $\left\langle\cup C u t_{j}, \cup I n t_{j}\right\rangle$ for updating $S \operatorname{core}(x)$ without accessing data records. For each value $c$ in $\operatorname{child}($ Best) added to $\left\langle\cup C u t_{j}, \cup I n t_{j}\right\rangle$ in the current iteration, we collect the following count statistics of $c$ while scanning data records in $P_{\text {Best }}$ for updating TIPS: (1) $\left|R_{c}\right|,\left|R_{d}\right|$, freq $\left(R_{c}, c l s\right)$, and $\operatorname{freq}\left(R_{d}, c l s\right)$ for computing InfoGain $(c)$, where $d \in$ child $(c)$ and $c l s$ is a class label. Refer to Section III for these notations. (2) $\left|P_{d}\right|$, where $P_{d}$ is a child partition under $P_{c}$ as if $c$ is refined, kept together with the leaf node for $P_{c}$. These information will be used in Section IV-D.

TIPS has several useful properties. (1) All data records in the same leaf partition have the same masked record although they may have different refined values. (2) Every data record appears in exactly one leaf partition. (3) Each leaf partition $P_{x}$ has exactly one masked $q i d_{j}$ on $Q I D_{j}$ and contributes the count $\left|P_{x}\right|$ towards $a\left(q i d_{j}\right)$. Later, we use the last property to extract $a\left(q i d_{j}\right)$ from TIPS.

Case 2: $D_{j}$ has no taxonomy tree. Consider a refinement $\perp_{j} \rightarrow\left\{\right.$ Best,$\left.\perp_{j}\right\}$ where $\perp_{j} \in D_{j}$, and $D_{j}$ is a categorical attribute without a taxonomy tree. First, we remove Best from $\operatorname{Sup}_{j}$. Then we replace $\perp_{j}$ with the disclosed value Best in all suppressed records that currently contain $\perp_{j}$ and originally contain Best. The TIPS data structure in Definition 3 can also support the refinement operation in this case. The only difference is to add an extra $L i n k_{\perp_{j}}$ to link up all leaf partitions $P_{\perp_{j}}$ containing value $\perp_{j}$. The candidate set now 


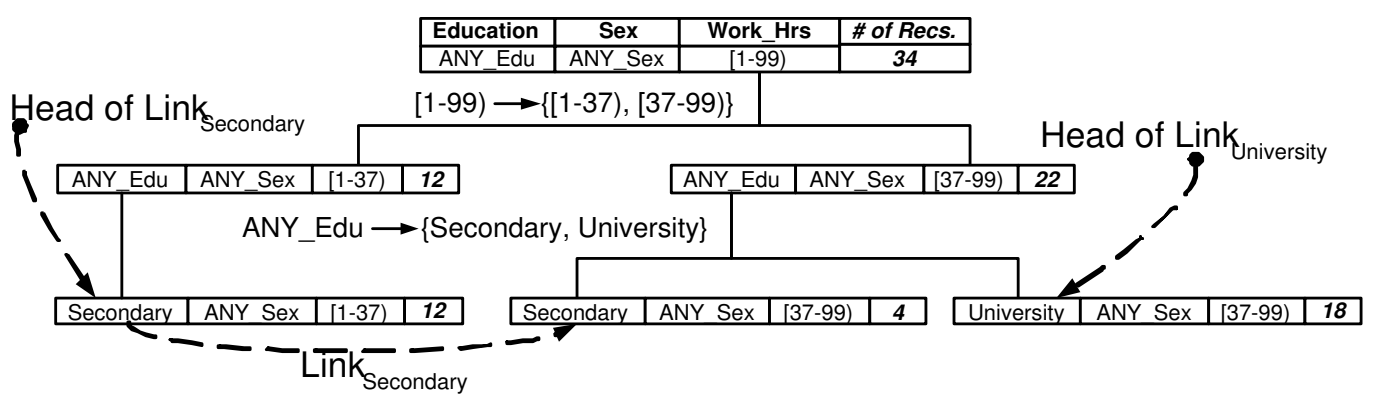

Fig. 3. The TIPS data structure

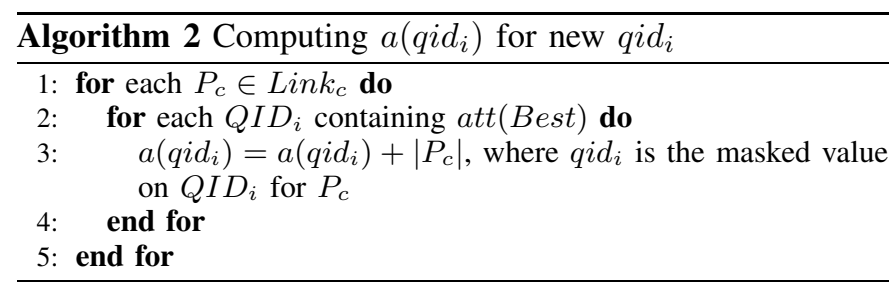

includes $\cup S u p_{j}$, that is, $\left\langle\cup C u t_{j}, \cup S u p_{j}, \cup I n t_{j}\right\rangle$.

Disclose Best in TIPS. We refine each leaf partition $P_{\perp_{j}}$ found on Link $_{\perp_{j}}$ as follows. Two child partitions $P_{\text {Best }}$ and $P_{\perp_{j}}^{\prime}$ are created under $P_{\perp_{j}}$. Data records in $P_{\perp_{j}}$ are split among the child partitions: $P_{\text {Best }}$ contains a data record $r$ in $P_{\perp_{j}}$ if Best is the original domain value in $r$; otherwise, $P_{\perp_{j}}^{\prime}$ contains $r$. Then link $P_{\text {Best }}$ to every $\operatorname{Link} k_{x}$ to which $P_{\perp_{j}}$ was previously linked, except for $L i n k_{\perp_{j}}$. Also, link $P_{\perp_{j}}^{\prime}$ to every $\operatorname{Link}_{x}$ to which $P_{\perp_{j}}$ was previously linked, except for Link Best. $_{\text {. }}$

Count statistics in TIPS. Similar to Case 1, we collect the following count statistics of $x \in \cup S u p_{j}$ while scanning data records in $P_{\perp_{j}}$ for updating TIPS. (1) $\left|R_{\perp_{j}}^{\prime}\right|,\left|R_{x}\right|$, freq $\left(R_{\perp_{j}}^{\prime}\right.$, cls $)$, freq $\left(R_{x}\right.$, cls $)$ for computing InfoGain $(x)$, where $x \in \cup S u p_{j}$ and cls is a class label. (2) $\left|P_{y}\right|$, where $P_{y}$ is a child partition under $P_{x}$ as if $x$ is disclosed, kept together with the leaf node for $P_{x}$. These information will be used in Section IV-D.

\section{Update Score and Validity (Line 6)}

This step updates $\operatorname{Score}(x)$ and validity for candidates $x$ in $\left\langle\cup C u t_{j}, \cup S u p_{j}, \cup I n t_{j}\right\rangle$ to reflect the impact of the Best refinement. The key is computing $\operatorname{Score}(x)$ from the count statistics maintained in Section IV-C without accessing data records. We update $\operatorname{InfoGain}(x)$ and $A_{x}\left(Q I D_{i}\right)$ separately. Note that the updated $A\left(Q I D_{i}\right)$ is obtained from $A_{\text {Best }}\left(Q I D_{i}\right)$.

1) Update InfoGain $(x)$ : An observation is that $\operatorname{InfoGain}(x)$ is not affected by Best $\rightarrow \operatorname{child}($ Best), except that we need to compute InfoGain $(c)$ for each newly added value $c$ in child(Best). InfoGain(c) can be computed while collecting the count statistics for $c$ in Case 1 of Section IV-C. In case that the refined attribute has no taxonomy tree, InfoGain $(x)$ can be computed from the count statistics for $x$ in Case 2 of Section IV-C.

2) Update AnonyLoss $(x)$ : Again, we consider the two cases:
Case 1: $D_{j}$ has a taxonomy tree. Unlike information gain, it is not enough to compute $A_{c}\left(Q I D_{i}\right)$ only for the new values $c$ in $\operatorname{child}($ Best $)$. Recall that $A_{x}\left(Q I D_{i}\right)$ is equal to the minimum $a\left(q i d_{i}\right)$ after refining $x$. If both $\operatorname{att}(x)$ and att(Best) are contained in $Q I D_{i}$, the refinement on Best may affect this minimum, hence, $A_{x}\left(Q I D_{i}\right)$. Below, we present a data structure, Quasi-Identifier TreeS (QITS), to extract $a\left(q i d_{i}\right)$ efficiently from TIPS for updating $A_{x}\left(Q I D_{i}\right)$.

Definition 4 (QITS): $Q I T_{i}$ for $Q I D_{i}=\left\{D_{1}, \ldots, D_{w}\right\}$ is a tree of $w$ levels. The level $p>0$ represents the masked values for $D_{p}$. Each root-to-leaf path represents an existing $q^{2} d_{i}$ on $Q I D_{i}$ in the masked data, with $a\left(q i d_{i}\right)$ stored at the leaf node. A branch is trimmed if its $a\left(q i d_{i}\right)=0$.

$A\left(Q I D_{i}\right)$ is equal to the minimum $a\left(q i d_{i}\right)$ in $Q I T_{i}$. In other words, $Q I T_{i}$ provides an index of $a\left(q i d_{i}\right)$ by $q i d_{i}$. Unlike TIPS, QITS does not maintain data records. On applying Best $\rightarrow \operatorname{child}\left(\right.$ Best), we update every $Q I T_{i}$ such that $Q I D_{i}$ contains the attribute att(Best).

Update $Q I T_{i}$. For each occurrence of Best in $Q I T_{i}$, create a separate branch for each $c$ in child(Best). The procedure in Algorithm 2 computes $a\left(q i d_{i}\right)$ for the newly created $q i d_{i}$ 's on such branches. The general idea is to loop through each $P_{c}$ on $\operatorname{Link}_{c}$ in TIPS, increment $a\left(q i d_{i}\right)$ by $\left|P_{c}\right|$. This step does not access data records because $\left|P_{c}\right|$ was part of the count statistics of Best. Let $r$ be the number of $Q I D_{i}$ containing att(Best). The number of $a\left(q i d_{i}\right)$ to be computed is at most $r \times \mid$ Link $_{\text {Best }}|\times| \operatorname{child}($ Best $) \mid$.

Example 8: In Figure 4, the initial $Q I T_{1}$ and $Q I T_{2}$ (i.e., left most) have a single path. After applying $[1-99) \rightarrow$ $\{[1-37),[37-99)\}$, the qid $\left\langle A N Y \_S e x,[1-99)\right\rangle$ in $Q I T_{2}$ is replaced with two new qids $\left\langle A N Y_{-}\right.$Sex, $\left.[1-37)\right\rangle$ and $\left\langle A N Y_{-} S e x,[37-99)\right\rangle$, and $A\left(Q I D_{2}\right)=12$. Since $Q I D_{1}$ does not include Work_Hrs, $Q I T_{1}$ remains unchanged and $A\left(Q I D_{1}\right)=34$.

After applying the second refinement $A N Y \_E d u \rightarrow$ $\{$ Secondary,University $\}, Q I T_{2}$ remains unchanged, and $A\left(Q I D_{2}\right)=12$. The qid $\left\langle A N Y_{-} E d u, A N Y_{-} S e x\right\rangle$ in $Q I T_{1}$ is replaced with two new qids $\left\langle\right.$ Secondary, $\left.A N Y \_S e x\right\rangle$ and $\left\langle\right.$ University, $\left.A N Y \_S e x\right\rangle$. To compute a(qid) for these new qids, we add $\left|P_{\text {Secondary }}\right|$ to $\operatorname{Link}_{\text {Secondary }}$ and $\left|P_{\text {University }}\right|$ to Link $k_{\text {University }}$ (see Figure 3): $a(\langle$ Secondary, ANY_Sex $\rangle)=0+12+4=16$, and $a\left(\left\langle\right.\right.$ University, $\left.\left.A N Y_{-} S e x\right\rangle\right)=0+18=18$. So $A_{A N Y_{-} E d u}\left(Q I D_{1}\right)=16$.

We now update $A_{x}\left(Q I D_{i}\right)$ for candidates $x$ in $\left\langle\cup C u t_{j}, \cup I n t_{j}\right\rangle$ (in the impact of Best $\rightarrow \operatorname{child}(B e s t)$ ). 


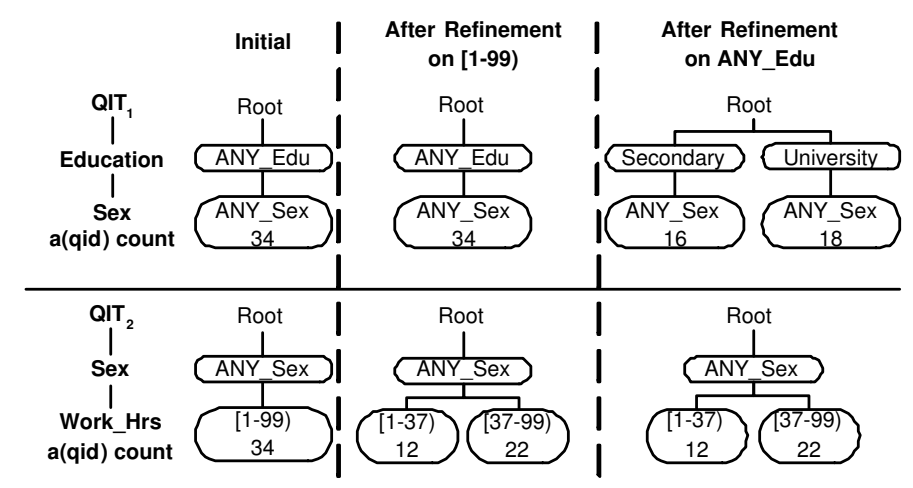

Fig. 4. The QITS data structure

Doing this by refining $x$ requires accessing data records, hence, is not scalable. We compute $A_{x}\left(Q I D_{i}\right)$ using the count statistics maintained for $x$ without accessing data records.

Update $A_{x}\left(Q I D_{i}\right)$. For a candidate $x$ in $\left\langle\cup C u t_{j}, \cup I n t_{j}\right\rangle$, computing $A_{x}\left(Q I D_{i}\right)$ is necessary in two cases. First, $x$ is in child(Best) because $A_{x}\left(Q I D_{i}\right)$ has not been computed for newly added candidates $x$. Second, $A_{x}\left(Q I D_{i}\right)$ might be affected by the refinement on Best, in which case att $(x)$ and att(Best) must be contained in $Q I D_{i}$. In both cases, we first compute $a\left(q i d_{i}^{x}\right)$ for the new $q i d_{i}^{x}$, s created as if $x$ is refined. The procedure is the same as in Algorithm 2 for refining Best, except that Best is replaced with $x$ and no actual update is performed on $Q I T_{i}$ and TIPS. Note that the count $\left|P_{c}\right|$, where $c$ is in $\operatorname{child}(x)$, used in the procedure is part of the count statistics maintained for $x$.

Next, we compare $a\left(q i d_{i}^{x}\right)$ with $A\left(Q I D_{i}\right)$ to determine the minimum, i.e., $A_{x}\left(Q I D_{i}\right)$. There are two subcases:

1) If no contributing qid of $A\left(Q I D_{i}\right)$ (i.e., $a($ qid $)=$ $\left.A\left(Q I D_{i}\right)\right)$ contains the value $x$, the contributing qids of $A\left(Q I D_{i}\right)$ will remain existing if $x$ is refined. Hence, $A_{x}\left(Q I D_{i}\right)$ is the minimum of $A\left(Q I D_{i}\right)$ and $a\left(q i d_{i}^{x}\right)$.

2) If some contributing qid of $A\left(Q I D_{i}\right)$ contains the value $x$, such qid's become new $q i d_{i}^{x}$ if $x$ is refined, so $A_{x}\left(Q I D_{i}\right)$ is the minimum of $a\left(q i d_{i}^{x}\right)$.

Finally, if the new $A_{x}\left(Q I D_{i}\right) \geq k_{i}$, we keep it with $x$ and mark $x$ as "valid" in the cut.

Case 2: $D_{j}$ has no taxonomy tree. Even the refined attribute has no taxonomy tree, the general operation of computing

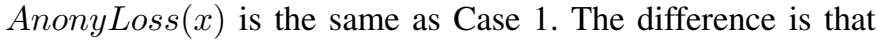
the refined values of $\perp_{j}$ becomes $\left\{\right.$ Best,$\left.\perp_{j}\right\}$ where Best is the disclosed value and the updated $\perp_{j}$ represents the remaining suppressed values $S u p_{j}$. Also, the candidate set includes $\cup S u p_{j}$, that is, $\left\langle\cup C u t_{j}, \cup S u p_{j}, \cup I n t_{j}\right\rangle$. On disclosing Best, we update all $Q I T_{i}$ such that att(Best) is in $Q I T_{i}$ to reflect the move of records from Link $_{\perp_{j}}$ to Link Best $_{\text {. }}$.

Update $Q I T_{i}$. For each occurrence of $\perp_{j}$ in $Q I T_{i}$, create a separate branch for Best and a separate branch for updated $\perp_{j}$. Follow the procedure in Algorithm 2 to compute $a\left(q i d_{i}\right)$ for the newly created $q i d_{i}$ 's on such branches, except that $P_{c}$ 's become $P_{\text {Best }}$ and $P_{\perp_{j}}^{\prime}$. Refer to Case 2 in Section IV-C for these notations.

\section{E. Efficiency Analysis}

Each iteration involves two types of work. The first type accesses data records in $R_{\text {Best }}$ or $R_{\perp_{j}}$ for updating TIPS and count statistics in Section IV-C. If Best is an interval, an extra step is required for determining the optimal split for each child interval $c$ in $\operatorname{child}($ Best). This requires making a scan on records in $R_{c}$, which is a subset of $R_{\text {Best }}$. To determine a split, $R_{c}$ has to be sorted which can be an expensive operation. Fortunately, resorting $R_{c}$ is unnecessary for each iteration because its superset $R_{\text {Best }}$ are already sorted. Thus, this type of work involves one scan of the records being refined in each iteration. The second type of work computes $\operatorname{Score}(x)$ for the candidates $x$ in $\left\langle\cup C u t_{j}, \cup S u p_{j}, \cup I n t_{j}\right\rangle$ without accessing data records in Section IV-D. For a table with $m$ attributes and each taxonomy tree with at most $p$ nodes, the number of such $x$ is at most $m \times p$. This computation makes use of the maintained count statistics and does not access data records. Let $h$ be the maximum number of times that a value in a record will be refined. For an attribute with a taxonomy tree, $h$ is bounded by the height of the taxonomy tree, and for an attribute without taxonomy tree, $h$ is bounded by 1 (i.e., a suppressed value is refined at most once). In the whole computation, each record will be refined at most $m \times h$ times, therefore accessed at most $m \times h$ times because only refined records are accessed. Since $m \times h$ is a small constant independent of the table size, our algorithm is linear in the table size.

In the special case that there is only a single QID, each rootto-leaf path in TIPS has represented a qid, and we can store $a($ qid) directly at the leaf partitions in TIPS without QITS. A single QID was considered in [7] [10] [12] [16] where the QID contains all potentially identifying attributes to be used for linking the table to an external source. Our algorithm is more efficient in this special case.

To focus on main ideas, our current implementation assumes that the compressed table fits in memory. Often, this assumption is valid because the compressed table can be much smaller than the original table. If the compressed table does not fit in the memory, we can store leaf partitions of TIPS on disk if necessary. Favorably, the memory is used to keep only leaf partitions that are smaller than the page size to avoid fragmentation of disk pages. A nice property of TDR is that leaf partitions that cannot be further refined (i.e., on which there is no candidate refinement) can be discarded, and only some statistics for them needs to be kept. This likely applies to small partitions in memory, therefore, the memory demand is unlikely to build up.

Compared to iteratively masking the data bottom-up starting from domain values, the top-down refinement is more natural and efficient for handling continuous attributes. To produce a small number of intervals for a continuous attribute, the top-down approach needs only a small number of interval splitting, whereas the bottom-up approach needs many interval merging starting from many domain values. In addition, the top-down approach can discard data records that cannot be further refined, whereas the bottom-up approach has to keep all data records until the end of computation. 


\section{EXPERIMENTAL EVALUATION}

Our goal in this section is to evaluate the proposed method, TDR, in terms of preserving the usefulness for classification and the scalability on large data sets. For the usefulness evaluation, we compare the classifier built from the masked data with the classifier built from the unmodified data. This comparison makes sense because the anonymization is due to the privacy consideration and the data will be released without modification in the absence of such consideration. In addition, the unmodified data has the lowest possible cost, therefore, serves the best possible candidate according to previous cost metrics [7] [12] [16]. Though some recent works such as [7] model the classification metric on the masked table, the optimality of such metrics does not translate into the optimality of classifiers, as pointed out in Section I. To our knowledge, [12] is the only work that has evaluated the impact of anonymity on classification with single dimensional generalization. For these reasons, our evaluation uses the baseline of the unmodified data and the reported results in [12]. All experiments on TDR were conducted on an Intel Pentium IV 2.6GHz PC with 1GB RAM.

\section{A. Data Quality}

Our first objective is to evaluate if the proposed TDR preserves the quality for classification while masking the data to satisfy various anonymity requirements. We used the C4.5 classifier [27] and Naive Bayesian classifier (from http://magix.fri.uni-lj.si/orange/) as classification models. We adopted three widely used benchmarks: Adult, Japanese Credit Screening, and German Credit Data were obtained from the UCI repository [28]. Unless stated otherwise, all attributes were used for building classifiers.

In a typical real life situation, the data provider releases all data records in a single file, leaving the split of training and testing sets to the data miner. Following this practice, we combined the training set and testing set into one set for masking, and built a classifier using the masked training set and collected the error using the masked testing set. This error, called the anonymity error, denoted $A E$, was compared with the baseline error, denoted $B E$, for the unmodified training and testing sets. Note that $A E$ depends on the anonymity requirement. $A E-B E$ measures the quality loss due to data masking.

\section{Data set: Adult}

The Adult data set has 6 continuous attributes, 8 categorical attributes, and a binary Class column representing two income levels, $\leq 50 \mathrm{~K}$ or $>50 \mathrm{~K}$. Table III(a) describes each attribute (cont. for continuous and cat. for categorical). After removing records with missing values from the pre-split training and testing sets, we have 30,162 records and 15,060 records for training and testing respectively. This is exactly the same data set as used in [12].

For the same anonymity threshold $k$, a single QID is always more restrictive than breaking it into multiple QIDs. For this reason, we first consider the case of single QID. To ensure that masking is working on attributes that have impact on classification, the QID contains the top $N$ attributes ranked
TABLE III

ATTRIBUTES FOR DATA SETS

(a) The Adult data set

\begin{tabular}{|l|l|l|l|}
\hline \multirow{2}{*}{ Attribute } & Type & \multicolumn{2}{|c|}{ Numerical Range } \\
\cline { 3 - 4 } & & \# of Leaves & \# of Levels \\
\hline Age (Ag) & cont. & $17-90$ \\
\hline Capital-gain (Cg) & cont. & $0-99999$ \\
\hline Capital-loss (Cl) & cont. & $0-4356$ \\
\hline Education-num (En) & cont. & $1-16$ \\
\hline Final-weight (Fw) & cont. & $13492-1490400$ \\
\hline Hours-per-week (H) & cont. & $1-99$ \\
\hline Education (E) & cat. & 16 & 5 \\
\hline Martial-status (M) & cat. & 7 & 4 \\
\hline Native-country (N) & cat. & 40 & 5 \\
\hline Occupation (O) & cat. & 14 & 3 \\
\hline Race (Ra) & cat. & 5 & 3 \\
\hline Relationship (Re) & cat. & 6 & 3 \\
\hline Sex (S) & cat. & 2 & 2 \\
\hline Work-class (W) & cat. & 8 & 5 \\
\hline
\end{tabular}

(b) The German data set

\begin{tabular}{|c|c|c|}
\hline Attribute & Type & $\begin{array}{c}\text { Numerical Range } \\
\text { \# of Values }\end{array}$ \\
\hline Duration $(\mathrm{Du})$ & cont. & $4-72$ \\
\hline Credit $(\mathrm{Cd})$ & cont. & $250-18424$ \\
\hline Installment-rate (Ir) & cont. & $1-4$ \\
\hline Residence-time (Rt) & cont. & $1-4$ \\
\hline Age $(\mathrm{Ag})$ & cont. & $19-75$ \\
\hline Existing-credits (Ec) & cont. & $1-4$ \\
\hline Liable-people (Li) & cont. & $1-2$ \\
\hline Account-status (As) & cat. & 4 \\
\hline Credit-history $(\mathrm{Ch})$ & cat. & 5 \\
\hline Loan-purpose (Lp) & cat. & 11 \\
\hline Savings-account (Sa) & cat. & 5 \\
\hline Employment (Em) & cat. & 5 \\
\hline Personal-status (Ps) & cat. & 5 \\
\hline Debtors (D) & cat. & 3 \\
\hline Property $(\mathrm{Pr})$ & cat. & 4 \\
\hline Installments (I) & cat. & 3 \\
\hline Housing $(\mathrm{H})$ & cat. & 3 \\
\hline Job (J) & cat. & 4 \\
\hline Telephone (T) & cat. & 2 \\
\hline Foreign $(\mathrm{F})$ & cat. & 2 \\
\hline
\end{tabular}

by the $\mathrm{C} 4.5$ classifier. The top rank attribute is the attribute at the top of the $\mathrm{C} 4.5$ decision tree. Then we remove this attribute and repeat this process to determine the rank of other attributes. The top 9 attributes are $C g, A g, M, E n, R e, H, S$, $E, O$ in that order. We specified three anonymity requirements denoted Top5, Top7, and Top9, where the QID contains the top 5, 7, and 9 attributes respectively. The upper error, denoted $U E$, refers to the error on the data with all the attributes in the QID removed (equivalent to generalizing them to the top most $A N Y$ or suppressing them to $\perp$ or including them into a full range interval). $U E-B E$ measures the impact of the QID on classification.

Figure 5 displays $A E$ for the $\mathrm{C} 4.5$ classifier with the anonymity threshold $20 \leq k \leq 1000$ by applying discretization on the 6 continuous attributes and suppression on the 8 categorical attributes without taxonomy trees. Note that $k$ is not spaced linearly. We summarize the analysis for Top7 as follows. First, $A E-B E$, where $B E=14.7 \%$, is less than $2.5 \%$ over the entire range of tested anonymity threshold, and $A E$ is much lower than $U E=21.5 \%$. This supports that accurate classification and privacy protection can coexist. Second, AE generally increases as the anonymity threshold $k$ increases, but not monotonically. For example, the error 


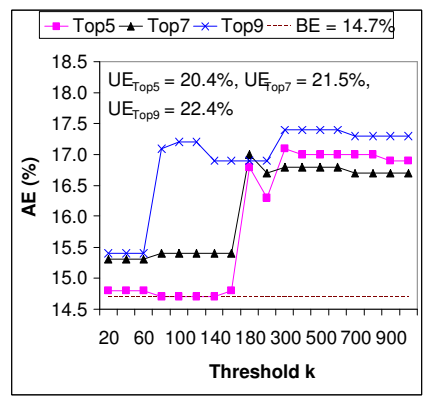

Fig. 5. Suppress and discretize TopN in Adult

slightly drops when $k$ increases from 180 to 200 . This is due to the variation between the training and testing sets, and the fact that a better structure may appear in a more masked state.

We further evaluate the effectiveness of generalization on categorical attributes with taxonomy trees. Although the author of [12] has specified taxonomy trees for categorical attributes, we do not agree with the author's groupings. For example, the author grouped Native-country according to continents, except Americas. We followed the grouping according to the World Factbook published by the CIA (http://www.cia.gov/cia/publications/factbook/).

Figure 6(a) displays $A E$ for the $\mathrm{C} 4.5$ classifier with the anonymity threshold $20 \leq k \leq 1000$ by applying discretization on the 6 continuous attributes and generalization on the 8 categorical attributes according to our specified taxonomy trees. We summarize the analysis for Top7 as follows. $A E-$ $B E$, where $B E=14.7 \%$, is less than $2 \%$ over the range of anonymity threshold $20 \leq k \leq 600$, and $A E$ is much lower than $U E=21.5 \%$. These results are similar to the results in Figure 5 although the finally masked versions of data are very different. This suggests there exists redundant "good" classification structures in the data.

A closer look at the masked data for Top7 with $k=500$ reveals that among the seven top ranked attributes, three are masked to a different degree of granularity, and four, namely $C g$ (ranked 1st), $A g$ (ranked 2nd), Re (ranked 5th), and $S$ (ranked 7th), are masked to the top most value $A N Y$. Even for this drastic masking, $A E$ has only increased by $2 \%$ from $B E=14.7 \%$, while the worst case can be $U E=21.5 \%$. With the masking, classification now is performed by the remaining three attributes in the $Q I D$ and the unmodified but lower ranked attributes. Clearly, this is a different classification structure from what would be found from the unmodified data. As a result, though masking may eliminate some structures, new structures emerge to help.

Figure 6(b) displays $A E$ for the Naive Bayesian classifier. Compared to the $\mathrm{C} 4.5$ classifier, though $B E$ and $U E$ are higher (which has to do with the classification method, not the masking), the quality loss due to masking, $A E-B E$ (note $B E=18.07 \%$ ), is smaller, no more than $1.5 \%$ for the range of anonymity threshold $20 \leq k \leq 1000$. This suggests that the information based masking is also useful to other classification methods such as the Naive Bayesian that do not use the information gain. Another observation is that $A E$ is even lower than $B E$ for the anonymity threshold $k \leq 180$ for Top5 and Top7. This confirms again that the optimal $k$ -

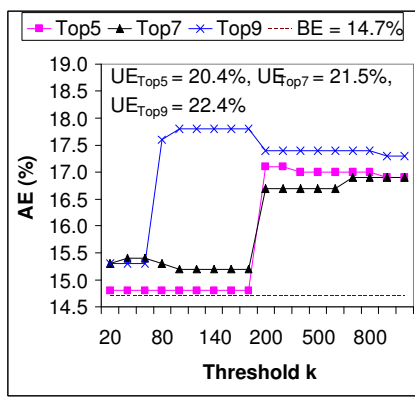

(a) $\mathrm{C} 4.5$

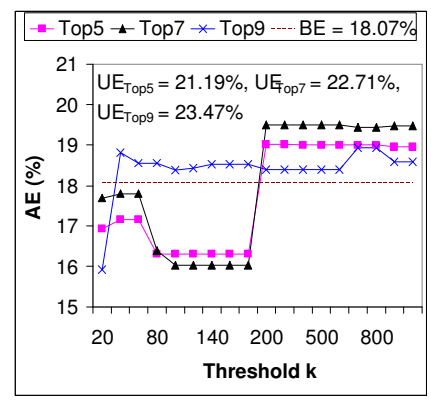

(b) Naive Bayesian
Fig. 6. Generalize and discretize TopN in Adult

anonymization is not relevant to the classification goal due to possibility of "over-fitting". The unmodified data certainly has the least distortion by any cost metric. However, this experiment shows that the least distortion does not translate into the accuracy of classifier. $A E<B E$ also occurs in the experiment on the $C R X$ data set in Figure 9(a). Our approach is bias toward masking the noises in order to help classification.
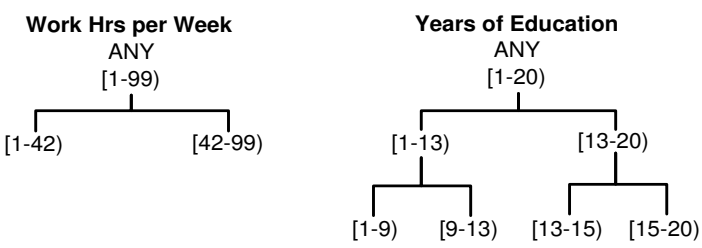

Fig. 7. Generated taxonomy trees of Hours-per-week and Education-num

Figure 7 shows the generated taxonomy trees for continuous attributes Hours-per-week and Education-num with Top7 and $k=60$. The splits are very reasonable. For example, in the taxonomy tree of Education-num, the split point at 13 distinguishes whether the person has post-secondary education. If the user does not like these trees, she may modify them or specify her own and subsequently treat continuous attributes as categorical attributes with specified taxonomy trees.

Our method took at most 10 seconds for all previous experiments. Out of the 10 seconds, approximately 8 seconds were spent on reading data records from disk and writing the masked data to disk. The actual processing time for generalizing the data is relatively short.

In an effort to study the effectiveness of multiple QIDs, we compared $A E$ between a multiple QIDs requirement and the corresponding single united QID requirement. We randomly generated 30 multiple QID requirements as follows. For each requirement, we first determined the number of QIDs using the uniform distribution $U[3,7]$ (i.e., randomly drawn a number between 3 and 7) and the length of QIDs using $U[2,9]$. For simplicity, all QIDs in the same requirement have the same length and same threshold $k=100$. For each QID, we randomly selected some attributes according to the QID length from the 14 attributes. A repeating QID was discarded. For example, a requirement of 3 QIDs and length 2 is $\{\langle\{A g, E n\}, k\rangle,\langle\{A g, R a\}, k\rangle$, $\langle\{S, H\}, k\rangle\}$, and the corresponding single QID requirement is $\{\langle\{A g, E n, R a, S, H\}, k\rangle\}$. 


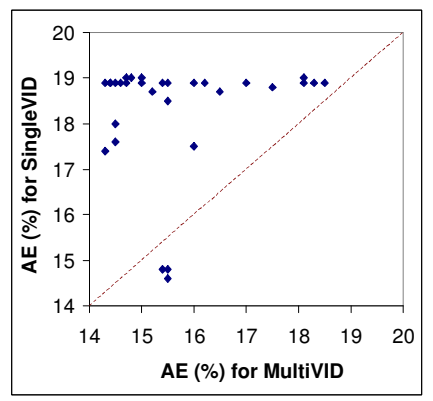

Fig. 8. SingleQID vs MultiQID $(k=100)$

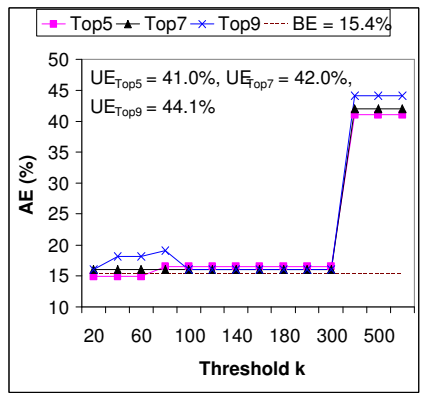

(a) $C R X$

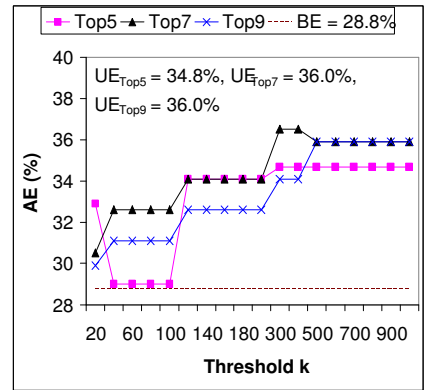

(b) German
Fig. 9. Suppress and discretize TopN in CRX and German

In Figure 8, each data point represents the $A E$ of a multiple QID requirement, denoted MultiQID, and the $A E$ of the corresponding single QID requirement, denoted SingleQID. The C4.5 classifier was used. Most data points appear at the upper left corner of the diagonal, suggesting that MultiQID generally yields lower $A E$ than its corresponding SingleQID. This verifies the effectiveness of multiple QIDs to avoid unnecessary masking and improve data quality.

\section{Data set: Japanese Credit Screening}

The Japanese Credit Screening data set, also known as $C R X$, is based on credit card application. There are 6 continuous attributes, 9 categorical attributes, and a binary class attribute representing the application status succeeded or failed. After removing records with missing values, there are 465 and 188 records for the pre-split training and testing respectively. In the UCI repository, all values and attribute names in $C R X$ have been changed to meaningless symbols, e.g., $A_{1} \ldots A_{15}$. No taxonomy tree is given in advance. The Top9 attributes in $C R X$ are $A_{9}, A_{11}, A_{10}, A_{8}, A_{15}, A_{7}, A_{14}, A_{6}, A_{5}$ in that order.

Figure 9(a) displays $A E$ for the $\mathrm{C} 4.5$ classifier with the anonymity threshold $20 \leq k \leq 600$ by applying discretization on the 6 continuous attributes and suppression on the 8 categorical attributes without taxonomy trees. Different anonymity requirements Top5, Top7, and Top9 yield similar $A E$ 's and similar patterns, indicating more restrictive requirement may not have much impact on classification quality. This largely depends on the availability of alternative "good" classification structures in the data set.

We summarize the analysis for Top7 as follows. First, $A E-$ $B E$, where $B E=15.4 \%$, is less than $4 \%$ over the range of anonymity threshold $20 \leq k \leq 300$, and $A E$ is much lower than $U E=42 \%$. This supports that accurate classification and privacy protection can coexist. $A E$ drastically increases when

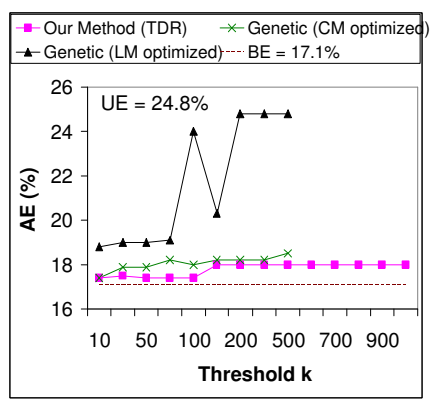

Fig. 10. Comparing with genetic algorithm

$k>300$ since $C R X$ only has 653 records.

\section{Data set: German Credit Data}

The German Credit Data, or simply German, has 7 continuous attributes, 13 categorical attributes, and a binary class attribute representing the good or bad credit risks. There are 666 and 334 records, without missing values, for the pre-split training and testing respectively. Table III(b) describes each attribute. The Top9 attributes in German are $C d, A s, D u$, $C h, S a, I, L p, D, P r$ in that order.

Figure 9(b) displays $A E$ for the $\mathrm{C} 4.5$ classifier with the anonymity threshold $20 \leq k \leq 1000$ by applying discretization on the 7 continuous attributes and suppression on the 13 categorical attributes without taxonomy trees. $A E-B E$, where $B E=28.8 \%$, is less than $4 \%$ over the range of anonymity threshold $20 \leq k \leq 100$ for the anonymity requirement Top7. Although $A E$ is mildly lower than $U E=$ $36 \%$, the benefit of masking $U E-A E$ is not as significant as in other data sets. If the data provider requires higher degree of anonymity, then she may consider simply removing the TopN attributes from the data set rather than masking them.

\section{B. Comparing with Other Algorithms}

[12] presented a genetic algorithm solution. This experiment was customized to conduct a fair comparison with the results in [12]. We used the same Adult data set, same attributes, and same anonymity requirement as specified in [12]:

$\mathrm{GA}=\langle\{A g, W, E, M, O, R a, S, N\}, k\rangle$.

We obtained the taxonomy trees from the author for generalization, except for the continuous attribute $A g$ which we used discretization. Following the procedure in [12], all attributes not in GA were removed and were not used to produce $B E$, $A E$, and $U E$ in this experiment, and all errors were based on the 10-fold cross validation and the $\mathrm{C} 4.5$ classifier. For each fold, we first masked the training data and then applied the masking to the testing data.

Figure 10 compares $A E$ of TDR with the errors reported for two methods in [12], Loss Metric (LM) and Classification Metric (CM), for $10 \leq k \leq 500$. TDR outperformed LM, especially for $k \geq 100$, but performed only slightly better than CM. TDR continued to perform well from $k=500$ to $k=1000$, for which no result was reported for LM and $\mathrm{CM}$ in [12]. This analysis shows that our method is at least comparable to genetic algorithm [12] in terms of accuracy. However, our method took only 7 seconds to mask the data, including reading data records from disk and writing the 


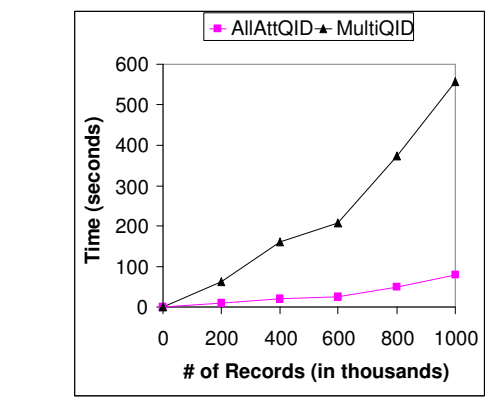

Fig. 11. Scalability vs \# of records $(k=50)$

masked data to disk. [12] reported that his method requires 18 hours to transform this data, which has about only $30 \mathrm{~K}$ data records. Clearly, the genetic algorithm is not scalable.

Recently, [17] compared with our previous version of TDR in [14] in terms of data quality on some other data sets. Their experiments suggested that the classification quality on the masked data can be further improved by using a more flexible masking operation, multidimensional generalization; however, this type of generalization suffers from the interpretation difficulty as discussed in Section I. [20] reported that the multidimensional generalization algorithm took about 10 seconds to mask the Adult data set. We compared TDR with some recently developed greedy anonymization algorithms that also conducted experiments on the Adult data set. The efficiency of the bottom-up cell generalization algorithm in [15] is comparable to TDR when $k=2,10$, but they did not report the efficiency for larger $k$. A cell generalization algorithm in [20] took about 60 seconds to mask the data. In general, multidimensional and cell generalization algorithms are less efficient than our method due to the larger number of possible masked tables.

\section{Efficiency and Scalability}

This experiment evaluates the scalability of TDR by blowing up the size of the Adult data set. First, we combined the training and testing sets, giving 45,222 records. For each original record $r$ in the combined set, we created $\alpha-1$ "variations" of $r$, where $\alpha>1$ is the blowup scale. For each variation of $r$, we randomly selected $q$ attributes from $\cup Q I D_{j}$, where $q$ has the uniform distribution $U\left[1,\left|\cup Q I D_{j}\right|\right]$, i.e., randomly drawn between 1 and the number of attributes in QIDs, and replaced the values on the selected attributes with values randomly drawn from the domain of the attributes. Together with all original records, the enlarged data set has $\alpha \times 45,222$ records. To provide a precise evaluation, the runtime reported excludes the time for loading data records from disk and the time for writing the masked data to disk.

Figure 11 depicts the runtime of TDR using generalization and discretization for $200 \mathrm{~K}$ to $1 \mathrm{M}$ data records and the anonymity threshold $k=50$ based on two types of anonymity requirements. AllAttQID refers to the single QID having all 14 attributes. This is one of the most time consuming settings because of the largest number of candidate refinements to consider at each iteration. For TDR, the small anonymity threshold of $k=50$ requires more iterations to reach a solution, hence more runtime, than a larger threshold. TDR takes approximately 80 seconds to transform $1 \mathrm{M}$ records.

In Figure 11, MultiQID refers to the average runtime over the 30 random multiple QID requirements in Section VA with $k=50$. Compared to AllAttQID, TDR becomes less efficient for handling multiple QIDs for two reasons. First, an anonymity requirement on multiple QIDs is a less restrictive constraint than the single QID anonymity requirement containing all attributes; therefore, TDR has to perform more refinements before violating the anonymity requirement. Moreover, TDR needs to create one QIT for each QID and maintains $a($ qid $)$ in QITS. The increase is roughly by a factor proportional to the number of QIDs in an anonymity requirement. The runtime of suppression and discretization on this expanded data set is roughly the same as shown in Figure 11.

\section{Summary}

Our experiments verified several claims about the proposed TDR method. First, TDR masks a given table to satisfy a broad range of anonymity requirements without sacrificing significantly the usefulness to classification. Second, while producing a comparable accuracy, TDR is much more efficient than previously reported approaches, particularly, the genetic algorithm in [12]. Third, the previous optimal $k$-anonymization [7] [16] does not necessarily translate into the optimality of classification. The proposed TDR finds a better anonymization solution for classification. Fourth, the proposed TDR scales well with large data sets and complex anonymity requirements. These performances together with the features discussed in Section I make TDR a practical technique for privacy protection while sharing information.

\section{Conclusions}

We considered the problem of ensuring individual's anonymity while releasing person-specific data for classification analysis. We pointed out that the previous optimal $k$ anonymization based on a closed form of cost metric does not address the classification requirement. Our approach is based on two observations specific to classification: information specific to individuals tends to be over-fitting, thus of little utility, to classification; even if a masking operation eliminates some useful classification structures, alternative structures in the data emerge to help. Therefore, not all data items are equally useful for classification and less useful data items provide the room for anonymizing the data without compromising the utility. With these observations, we presented a top-down approach to iteratively refine the data from a general state into a special state, guided by maximizing the trade-off between information and anonymity. This top-down approach serves a natural and efficient structure for handling categorical and continuous attributes and multiple anonymity requirements. Experiments showed that our approach effectively preserves both information utility and individual's privacy and scales well for large data sets. 


\section{REFERENCES}

[1] P. Samarati and L. Sweeney, "Generalizing data to provide anonymity when disclosing information," in Proc. of the 17th ACM SIGACTSIGMOD-SIGART Symposium on Principles of Database Systems (PODS 98), Seattle, WA, June 1998, p. 188.

[2] P. Samarati, "Protecting respondents' identities in microdata release," in IEEE Transactions on Knowledge Engineering (TKDE), vol. 13, no. 6, 2001, pp. 1010-1027.

[3] The House of Commons in Canada, "The personal information protection and electronic documents act," April 2000, http://www.privcom.gc.ca/.

[4] S. M. Weiss and C. A. Kulikowski, Computer Systems That Learn: Classification and Prediction Methods from Statistics, Machine Learning, and Expert Systems. San Mateo, CA: Morgan Kaufmann, 1991.

[5] T. Dalenius, "Finding a needle in a haystack - or identifying anonymous census record," Journal of Official Statistics, vol. 2, no. 3, pp. 329-336, 1986.

[6] L. Sweeney, "Achieving $k$-anonymity privacy protection using generalization and suppression," International Journal on Uncertainty, Fuzziness, and Knowledge-based Systems, vol. 10, no. 5, pp. 571-588, 2002.

[7] R. J. Bayardo and R. Agrawal, "Data privacy through optimal kanonymization," in Proc. of the 21st International Conference on Data Engineering (ICDE), Tokyo, Japan, April 2005, pp. 217-228.

[8] G. Aggarwal, T. Feder, K. Kenthapadi, R. Motwani, R. Panigrahy, D. Thomas, and A. Zhu, "Approximation algorithms for k-anonymity," Journal of Privacy Technology, no. 20051120001, November 2005.

[9] A. Meyerson and R. Williams, "On the complexity of optimal kanonymity," in Proc. of the 23rd ACM Symposium on Principles of Database Systems (PODS), 2004, pp. 223-228.

[10] L. Sweeney, "Datafly: A system for providing anonymity in medical data," in Proc. of the International Conference on Database Security, 1998, pp. 356-381.

[11] A. Hundepool and L. Willenborg, " $\mu$ - and $\tau$-argus: Software for statistical disclosure control," in Proc. of the 3rd International Seminar on Statistical Confidentiality, Bled, 1996.

[12] V. S. Iyengar, "Transforming data to satisfy privacy constraints," in Proc. of the 8th ACM SIGKDD International Conference on Knowledge Discovery and Data Mining, Edmonton, AB, Canada, July 2002, pp. 279-288.

[13] K. Wang, P. Yu, and S. Chakraborty, "Bottom-up generalization: a data mining solution to privacy protection," in Proc. of the 4th IEEE International Conference on Data Mining (ICDM), November 2004.

[14] B. C. M. Fung, K. Wang, and P. S. Yu, "Top-down specialization for information and privacy preservation," in Proc. of the 21 st International Conference on Data Engineering (ICDE), Tokyo, Japan, April 2005, pp. 205-216.

[15] R. C. W. Wong, J. Li., A. W. C. Fu, and K. Wang, " $(\alpha, k)$-anonymity: An enhanced $k$-anonymity model for privacy preserving data publishing," in Proc. of the 12th ACM SIGKDD International Conference on Knowledge Discovery and Data Mining (KDD), Philadelphia, PA, August 2006.

[16] K. LeFevre, D. J. DeWitt, and R. Ramakrishnan, "Incognito: Efficient full-domain k-anonymity," in Proc. of the 2005 ACM SIGMOD International Conference on Management of Data, 2005, pp. 49-60.

[17] — , "Workload-aware anonymization," in Proc. of the 12th ACM SIGKDD International Conference on Knowledge Discovery and Data Mining (KDD), Philadelphia, PA, August 2006.

[18] K. Wang, B. C. M. Fung, and G. Dong, "Integrating private databases for data analysis," in Proc. of the 2005 IEEE International Conference on Intelligence and Security Informatics (ISI), Atlanta, GA, May 2005, pp. 171-182.

[19] K. LeFevre, D. J. DeWitt, and R. Ramakrishnan, "Mondrian multidimensional k-anonymity," in Proc. of the 22nd IEEE International Conference on Data Engineering (ICDE), Atlanta, GA, 2006.

[20] J. Xu, W. Wang, J. Pei, X. Wang, B. Shi, and A. W. C. Fu, "Utilitybased anonymization using local recoding," in Proc. of the 12th ACM SIGKDD International Conference on Knowledge Discovery and Data Mining (KDD), Philadelphia, PA, August 2006.

[21] A. Machanavajihala, J. Gehrke, and D. Kifer, "l-diversity: Privacy beyond $k$-anonymity," in Proc. of the 22nd International Conference on Data Engineering (ICDE), 2006.

[22] K. Wang, B. C. M. Fung, and P. S. Yu, "Template-based privacy preservation in classification problems," in Proc. of the 5th IEEE International Conference on Data Mining (ICDM), Houston, TX, November 2005, pp. $466-473$.
[23] — - "Handicapping attacker's confidence: An alternative to kanonymization," Knowledge and Information Systems: An International Journal (KAIS), 2006.

[24] X. Xiao and Y. Tao, "Personalized privacy preservation," in Proc. of the 2006 ACM SIGMOD International Conference on Management of Data, June 2006.

[25] K. Wang and B. C. M. Fung, "Anonymizing sequential releases," in Proc. of the 12th ACM SIGKDD International Conference on Knowledge Discovery and Data Mining (KDD), Philadelphia, PA, August 2006, pp. 414-423.

[26] C. E. Shannon, "A mathematical theory of communication," The Bell System Technical Journal, vol. 27, p. 379 and 623, 1948.

[27] J. R. Quinlan, C4.5: Programs for Machine Learning. Morgan Kaufmann, 1993

[28] D. J. Newman, S. Hettich, C. L. Blake, and C. J. Merz, "UCI repository of machine learning databases," 1998. [Online]. Available: http://www.ics.uci.edu/ mlearn/MLRepository.html

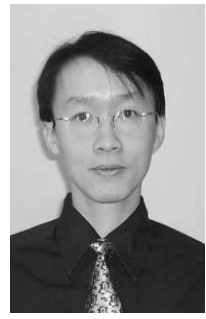

Benjamin C. M. Fung received B.Sc. and M.Sc. degrees in computing science from Simon Fraser University. $\mathrm{He}$ is currently a Ph.D. candidate at Simon Fraser with the postgraduate scholarship doctoral award from the Natural Sciences and Engineering Research Council of Canada (NSERC). His recent research interests include privacy-preserving data mining/publishing, secure distributed computing, and text mining. Before pursuing his Ph.D., he worked in the R\&D department at Business Objects and designed reporting systems for various ERP and CRM systems. Mr. Fung has published in data mining and security conferences, journals, and books. He has served program committees for international conferences.

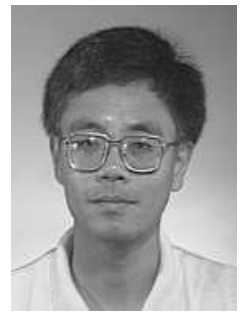

Ke Wang received Ph.D. from Georgia Institute of Technology. He is currently a professor at School of Computing Science, Simon Fraser University. Before joining Simon Fraser, he was an associate professor at National University of Singapore. He has taught in the areas of database and data mining. Dr. Wang's research interests include database technology, data mining and knowledge discovery, machine learning, and emerging applications, with recent interests focusing on the end use of data mining. This includes explicitly modelling the business goal (such as profit mining, bio-mining and web mining) and exploiting user prior knowledge (such as extracting unexpected patterns and actionable knowledge). He is interested in combining the strengths of various fields such as database, statistics, machine learning and optimization to provide actionable solutions to real life problems. He is an associate editor of the IEEE TKDE journal and has served program committees for international conferences.

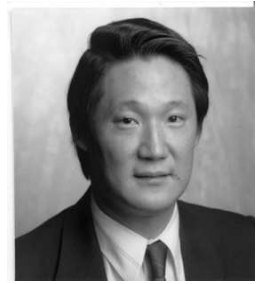

Philip S. Yu received B.S. Degree in E.E. from National Taiwan University, M.S. and Ph.D. degrees in E.E. from Stanford University, and M.B.A. degree from New York University. He is with IBM T.J. Watson Research Center and currently manager of the Software Tools and Techniques group. Dr. Yu has published more than 450 papers in refereed journals and conferences. He holds or has applied for more than 250 US patents. Dr. Yu is a Fellow of the ACM and the IEEE. He has received several IBM honors including 2 IBM Outstanding Innovation Awards, an Outstanding Technical Achievement Award, 2 Research Division Awards and the 85th plateau of Invention Achievement Awards. He received a Research Contributions Award from IEEE Intl. Conference on Data Mining in 2003 and also an IEEE Region 1 Award for "promoting and perpetuating numerous new electrical engineering concepts" in 1999. Dr. Yu is an IBM Master Inventor. 